\title{
Viable production mechanism of keV sterile neutrino with large mixing angle
}

\author{
A. Chudaykin $1,2, a$ \\ ${ }^{1}$ Institute for Nuclear Research of the Russian Academy of Sciences, 60th October Anniversary prospect \\ 7a, Moscow 117312, Russia \\ ${ }^{2}$ Moscow Institute of Physics and Technology, Institutsky per. 9, Dolgoprudny 141700, Russia
}

\begin{abstract}
We study a model with a hidden sector coupled to keV scale sterile neutrinos. Due to nontrivial dynamics of this sector, the initially massless sterile neutrino acquires a nonzero mass at some temperature corresponding to the phase transition in the hidden sector. It shifts the onset of oscillations in plasma to later times, so that the final abundance of sterile neutrinos is strongly suppressed. We argue that in this model various cosmological and astrophysical bounds can be significantly alleviated opening new perspectives for ground-based experiments such as Troitsk $v$-mass and KATRIN in the large mixing region.
\end{abstract}

\section{Introduction}

Introduction of sterile neutrinos is one of the most popular ways to minimally extend the Standart Model (SM) of particle physics [1]. For the mass range of sterile neutrinos $1-100 \mathrm{keV}$, the mixing between active and sterile neutrino states should be sufficiently small, $\sin ^{2}(2 \theta) \ll 10^{-7}$. Indeed, the active neutrinos are effectively produced via oscillations in the early Universe and can easily overproduce the DM today [2,3]. In addition, cosmic X-ray observations which measure monochromatic photons which can be gained through a radiative decay mode of sterile neutrinos, $N \rightarrow \gamma v$, can impose an even stronger constraint on the mixing angle [4-6]. Finally, there are fairly weak bounds from direct searches, $\sin ^{2}(2 \theta)<10^{-3}-10^{-2}$, valid for any sterile neutrino abundance.

We argue that the sterile neutrino production via oscillations in some phenomenological models can be significantly suppressed. Such dumping depends only on specific properties of the hidden sector. In the early Universe the most effective generation of sterile neutrinos through the activesterile mixing happens at

$$
T_{\max } \sim 133 \mathrm{MeV}\left(\frac{M}{1 \mathrm{keV}}\right)^{1 / 3} .
$$

So, if oscillations start at $T \sim T_{c} \ll T_{\max }$, where $T_{c}$ denotes the critical temperature of the onset of scalar field oscilations, the present abundance of sterile neutrinos will be depleted.

We will stick to the following plan. In section 2 we calculate the sterile neutrino abundance and velocity distribution in the case when oscillations are available only at $T \lesssim T_{c}$. Then, in section 3 , we

\footnotetext{
a e-mail: chudy@ms2.inr.ac.ru
} 
discuss how to suspend the onset of neutrino oscillations and introduce a changing in time sterile neutrino mass (see different models with the variable neutrino masses [7, 8]). Finally, in section 4, outputs and available regions in parameter space $\theta^{2}-M$ compatible with all cosmological and astrophysical bounds within the proposed framework are presented.

\section{Generation of a variable mass sterile neutrino}

We calculate the generation of sterile neutrino in the model

$$
\mathcal{L}=i \bar{N} \hat{\partial} N+\frac{M}{2} \bar{N}^{c} N+y_{v} H \bar{v}_{a} N+\text { h.c. }
$$

where the Yukawa term gives the Dirac mass $m_{D}=y_{v}\langle H\rangle$ yielding the active neutrino mass $m=\theta^{2} M$ and the active-sterile mixing angle $\theta \simeq m_{D} / M$. Such a mixing provides the effective production of sterile neutrinos in the hot plasma. The analytic approach developed in [2] assumes that the total number of relativistic degrees of freedom $g_{*}$ changes insignificantly during the oscillation process. In our case we are interested in the temperatures $T \ll 100 \mathrm{MeV}$, so that one can treat $g_{*}$ as a constant value ${ }^{1}$. Moreover, we assume that the active neutrino number does not decrease dramatically by conversion to the sterile neutrinos. It is valid for small enough mixing angles both above and below the freeze-out of active neutrinos occurred at $T_{v, f} \sim 2 \mathrm{MeV}$. Under these assumptions, the distribution function of sterile neutrinos obeys the following equation

$$
H T\left(\frac{\partial f_{N}}{\partial T}\right)_{E / T}=\left[\frac{1}{2} \sin ^{2}\left(2 \theta_{M}\right)\right] \frac{\Gamma_{A}}{2} f_{A},
$$

where the effective angle and interaction rate of active neutrino scattering in the plasma assuming the mixing between electronic and sterile neutrinos ${ }^{2}$ read

$$
\sin ^{2}\left(2 \theta_{M}\right)=\frac{m_{D}^{2}}{m_{D}^{2}+\left[c \Gamma_{A} E / M+M / 2\right]^{2}}, \quad \Gamma_{A} \approx 1.27 \times G_{F}^{2} T^{4} E,
$$

where $c \approx 63$ [9]. The solution of (3) can be explicitly found in the following form

$$
\frac{f_{N}}{f_{A}}=\frac{2.9}{g_{*}^{1 / 2}}\left(\frac{\theta^{2}}{10^{-6}}\right)\left(\frac{M}{\mathrm{keV}}\right) \int_{x}^{x_{c}} \frac{y d x^{\prime}}{\left(1+y^{2} x^{\prime 2}\right)^{2}},
$$

where $y \equiv E / T, x \equiv 148(T / \mathrm{GeV})^{3}(\mathrm{keV} / M)$ with $T$ is the current temperature under consideration and $T_{c}$ - the critical temperature. $f_{A}(y)=1 /\left(\mathrm{e}^{y}+1\right)$ is the distribution function of the active neutrinos and $g_{*}=10.75$ is relativistic degrees of freedom just before the freeze-out of active neutrinos. We can put the lower integration bound to $x=0$ and expand the right side of expression (5) in the limit $x_{c} \ll 1$ assuming $T_{c} \ll 100 \mathrm{MeV}$ that yields the following sterile neutrino distribution

$$
\frac{f_{N}(y)}{f_{A}(y)} \simeq 0.13 \times \theta^{2}\left(\frac{10.75}{g_{*}}\right)^{1 / 2}\left(\frac{T_{c}}{\mathrm{MeV}}\right)^{3} \cdot y .
$$

\footnotetext{
${ }^{1}$ In fact, in what follows we express all quantities in terms of the active neutrino temperature $T_{v}$, so that we avoid difficulties with rapid changing $g_{*}$ after the active neutrino decoupling.

${ }^{2}$ The developed here approach can be applied to muon and tau neutrinos as well with an appropriate modification of numerical factors in $\Gamma_{A}$ and $c$. For more detailed information, see [9]
} 
The obtained dependence over $y$ dictates a warm spectrum with average momentum $\langle\langle p\rangle\rangle=4.1 T$ different from the usual relation $\langle\langle p\rangle\rangle=3.1 T$ in case of non-resonant production. The number density for distribution law (6) leads to the following sterile neutrino contribution to the total energy density of Universe today

$$
h^{2} \Omega_{N} \simeq 4.3 \times \theta^{2}\left(\frac{10.75}{g_{*}}\right)^{1 / 2}\left(\frac{T_{c}}{\mathrm{MeV}}\right)^{3}\left(\frac{M}{\mathrm{keV}}\right)
$$

which is strongly suppresed provided that $T_{c} \ll 100 \mathrm{MeV}$. In Fig. 1 the results of numerical integration of (3) considering proper temperature dependencies of $g_{*}$ and $\Gamma_{A}$ taken from refs. [10,11] are shown. These plots exhibit the applicability of (7) in the interesting range $T_{c} \ll 100 \mathrm{MeV}$ and reveal the right behaviors in case $T_{c} \sim T_{\max }$.
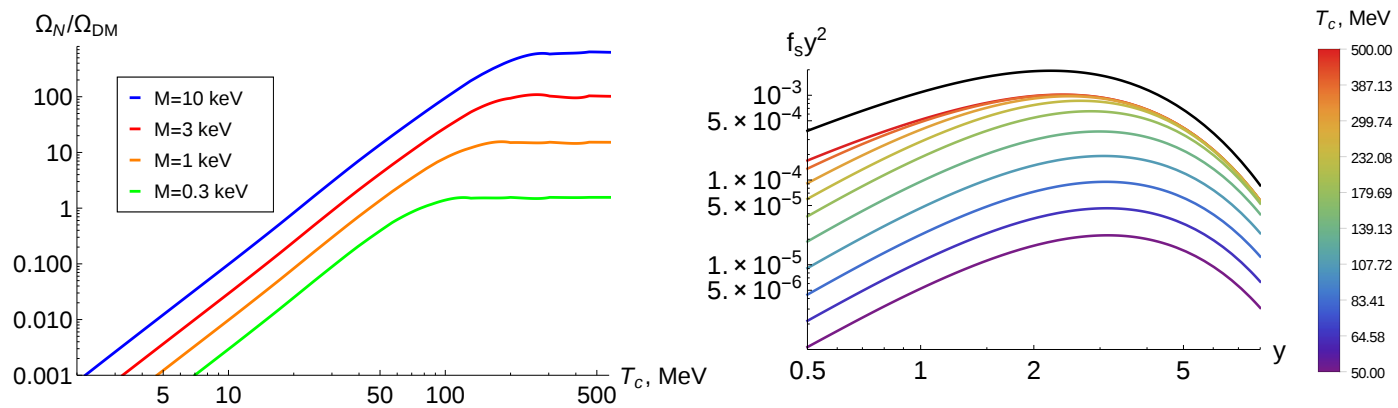

Figure 1. Left panel: Evolution of energy density fraction of sterile neutrinos in whole DM produced in oscillations at $T<T_{c}$ for different values of critical temperature $T_{c}$ and several masses $M$ of sterile neutrinos. Right panel: momentum distribuion of sterile neutrinos for one sterile neutrino reference mass $M=7.1 \mathrm{keV}$; top black curve depict the thermal Fermi-Dirac distribution. For the both panels $\sin ^{2}(2 \theta)=10^{-6}$ is employed.

Remarkably, neutrino oscillations with a relatively large mixing provides an appropriate values of active neutrino mass within the seesaw type I mechanism [12-16]. Such situation is oppisite to non-resonant or resonant Shi-Fuller production mechanisms (see ref. [17] for details). Indeed, typical values of the mixing angle explaining the small active neutrino masses is given by

$$
\frac{\theta^{2}}{10^{-4}}=\frac{1 \mathrm{keV}}{M} \frac{m}{0.1 \mathrm{eV}}
$$

For solar or atmospheric neutrinos the typical masses are $\sqrt{\delta m_{\mathrm{sol}}^{2}} \simeq 0.9 \times 10^{-2} \mathrm{eV}$ or $\sqrt{\delta m_{\mathrm{atm}}^{2}} \simeq$ $0.05 \mathrm{eV}$, respectiviely. Thus, if $T_{c} \sim \mathrm{MeV}$ the present sterile neutrino abundance described by (7) is significantly small, $\Omega_{N} \ll 1$, to meet the DM overproduction and X-ray constraints (see section 4 for details) and the $\mathrm{keV}$-scale sterile neutrinos are capable to explain active neutrino masses within the seesaw type I mechanism.

\section{Phase transition in the hidden sector}

To explain active neutrino masses within the framework of the classic see-saw mechanism one need a relatively large mixing (8), for that the transitions from active neutrinos into sterile ones in the early Universe should be effectively suppressed. It can be achieved in the case when the sterile neutrino is 
treated initially massless. In addition, we introduce the hidden sector coupled to the sterile neutrinos in such a way that the mass of the sterile neutrino is defined by the value of the singlet scalar field. It can be achieved by introducing the late-time phase transition in the hidden sector when at some moment of time the scalar field acquires the non-zero vacuum expectation value and the sterile neutrinos gain the Majorana mass. In this scenario, the oscillations turn out to be blocked before the phase transition in the hidden sector when sterile neutrinos are massless. We also assume that the hidden sector was in thermal equilibrium with the SM particles such a long time ago that its own temperature is much smaller than one of the SM plasma, or $T_{h}=\xi T$, with $\xi \ll 1$. It enables us to not consider the hidden sector contribution to the energy density of the Universe. We employ the most straightforward idea that the mass of sterile neutrinos is defined through a Yukawa type interaction

$$
\mathcal{L}=\frac{f}{2} \phi \bar{N}^{c} N+\text { h.c. }
$$

that gives the Majorana mass of the fermions $M=f\langle\langle\phi\rangle\rangle$ after the phase transition.

Under requirements discussed above the strong dumping of oscillations takes place when the field $\phi$ is in the symmetric state at high temperatures

$$
\left.\langle\phi \phi\rangle\right|_{T_{h}>\xi T_{c}}=0,
$$

and acquirs the vacum expectation value at lower temperatures,

$$
\left\langle\left.\langle\phi\rangle\right|_{T_{h}<\xi T_{c}}=v_{\phi}, \quad M=f v_{\phi} .\right.
$$

Assuming the instant phase transition, the spectrum of sterile neutrinos produced in oscillations at $T \lesssim T_{c}$ is given by (5) and the present abundance of them - by (7).

We find that the sterile neutrino abundance (7) produced at $T \lesssim T_{c}$ can be significantly reduced by lowering the temperature of phase transition $T_{c}$. Let us see what is the minimum number of sterile neutrinos we can achieve in such a model. In (6) we assume that the sterile neutrinos are absent in the Universe at $T_{c}$. Actually, since the Majorana mass equals 0 , the sterile neutrino acts as the pure Dirac fermion of mass $m_{D}$. Thus, one expects the admixture of right-handed particles produced in weak scattering at the level of $m_{D}^{2} / 4 p^{2}=m_{D}^{2} / 4 y^{2} T^{2}$. Therefore, in addition to (6) we expect the initial contribution to the overall momentum distribution of sterile neutrinos

$$
\frac{f_{S, \text { in }}}{f_{A}} \simeq \frac{m_{D}^{2}}{4 y^{2} T_{c}^{2}} \simeq \frac{0.25 \times 10^{-6} \theta^{2}}{y^{2}}\left(\frac{M}{\mathrm{keV}}\right)^{2}\left(\frac{\mathrm{MeV}}{T_{c}}\right)^{2},
$$

where we used $m_{D}^{2}=\theta^{2} M^{2}$. This spectrum generates the additional abundance of sterile neutrinos today

$$
h^{2} \Omega_{N, \text { in }}=\frac{M T_{v, 0}^{3}}{\left(\rho_{c} / h^{2}\right)} \frac{2}{2 \pi^{2}} \int_{0}^{\infty} d y y^{2} f_{S, \text { in }}(y) \approx 10^{-6} \theta^{2}\left(\frac{M}{\mathrm{keV}}\right)^{3}\left(\frac{\mathrm{MeV}}{T_{c}}\right)^{2} .
$$

Since initial (11) and the late (7) sterile neutrino abundances have the opposite dependencies over $T_{c}$, they become equal at ${ }^{3}$

$$
T_{c, \text { min }} \simeq 0.05 \mathrm{MeV}\left(\frac{M}{\mathrm{keV}}\right)^{2 / 5} .
$$

So, the minimal sterile neutrino abundance is saturated by (7) and (11) contributions at critical temperature (12)

$$
h^{2} \Omega_{N, \min } \simeq h^{2} \Omega_{N, T<T_{C}}+h^{2} \Omega_{N, \text { in }} \simeq 0.9 \times 10^{-3} \theta^{2}\left(\frac{M}{\mathrm{keV}}\right)^{11 / 5} .
$$

\footnotetext{
${ }^{3}$ We exploited here $g_{*} \approx 13$ that is valid for the phase transition temperature of active neutrinos $T_{c}<1 \mathrm{MeV}$.
} 
The final spectrum of sterile neutrinos is given by the sum of generated (6) and initial (10) contributions, that is $f_{N}=f_{S, T<T_{C}}+f_{S \text {,in }}$. The former spectrum is warm in comparison with the thermal Fermi-Dirac distribution whereas the latter one is cold. Thus, the initial contribution may help to avoid structure formation bounds on the DM. However, such effect proves to be not so prominent and does not help to constitute all the DM today as will be explained in Sec. 4.

We do not provide here the concrete realisation of the hidden sector but list several requirements which help the reader to formulate such a theory. Firstly, the phase transition should be fast enough. Secondly, it will be more preferably if interactions in the hidden sector are in the weak coupling regime. And finally, the Yukawa term (9) should not destroy the usual oscillation picture, so the rate of sterile neutrino scattering in the hidden sector must be much smaller than $\Gamma_{A}$.

\section{Discussion of cosmological and astrophysical constraints}

We consider the present status of cosmological and astrophysical constraints in the light of one sterile neutrino production mechanism proposed in Sec. 3. Then, we confront the available region obtained in this model to projected sensitivities of different ground-based experiments.

We regard there three main sources of constraints coming from cosmology and astrophysics. The first bound originates from the requirement that sterile neutrinos do not overproduce the DM, so $\Omega_{N} \leq \Omega_{\mathrm{DM}}$.

The second constraint corresponds to a radiative decay mode of the sterile neutrino $N \rightarrow \gamma v$ that produces the monochromic photons of energy $E=M / 2$. X-ray observations put the upper bound on this photon flux at a given energy and severely restrict the active-sterile mixing, see [17]. We denote the maximum available mixing angle provided by $\mathrm{X}$-ray experiments by $\theta_{\mathrm{X} \text {-ray }}(M)$ assuming that sterile neutrinos compose all the DM today. In the case when sterile neutrinos constitute only a part of DM, the actual bound should be rescaled as follows

$$
\sin ^{2} 2 \theta(M)<\frac{\Omega_{\mathrm{DM}}}{\Omega_{N}} \sin ^{2} 2 \theta_{\mathrm{X} \text {-ray }}(M) .
$$

Third, if sterile neutrinos contribute a significant part to the DM $\Omega_{N} \lesssim \Omega_{\mathrm{DM}}$, one should account for the structure formation bounds arriving from the spacial distribution of dwarfs and small satellite galaxies, the phase space density of dark matter in galaxies and the absorption spectrum in Ly- $\alpha$ clouds. All these bounds constrain the level of free-streaming limiting the average velocity of the dark matter particles. Indeed, fastly enough spreading particles effectively erase the underlying density field on small scales.

We found the accurate numerical solutions of (3) with a help of (10) exploiting proper temperature dependencies of $g_{*}$ and $\Gamma_{A}$ taken from Ref. [10,11]. In the developed approach we show in Fig. 2 all actual constraints in the scenario of section 3. These plots represent the maximal available sterile neutrino fraction in the total DM abundance, $\Omega_{N} / \Omega_{\mathrm{DM}}$, and maximal available phase transition temperature $T_{c}$ which address all cosmological and astrophysical constraints properly.

We shortly list below all bounds relevant to the usual non-resonant production. The black line in Fig. 2 depicts the mixing angle $\sin ^{2} 2 \theta_{\mathrm{NRP}}$ that corresponds to the non-resonant generation of the total DM by sterile neutrinos. The blue line depicts the X-ray constraints $\theta_{\mathrm{X} \text {-ray }}$ assuming $\Omega_{N}=\Omega_{\mathrm{DM}}$. In the region $\theta_{\mathrm{X} \text {-ray }}<\theta_{\mathrm{NRP}}$ the actual X-ray constraint is shown by orange line and defined in the different way

$$
\theta_{\max X-\text { ray }}^{2}(M)=\sqrt{\theta_{\mathrm{X} \text {-ray }}^{2}(M) \theta_{\mathrm{NRP}}^{2}(M)} .
$$

Really, the amount of sterile neutrinos produced in this region via the non-resonant production is small, and one should rescale the usual X-ray bound $\theta_{\mathrm{X} \text {-ray }}$ for the case $\Omega_{N}<\Omega_{\mathrm{DM}}$ according to (15). 
Now we refer to the particular regions of the plots in Fig. 2 to highlight the underlying physics.
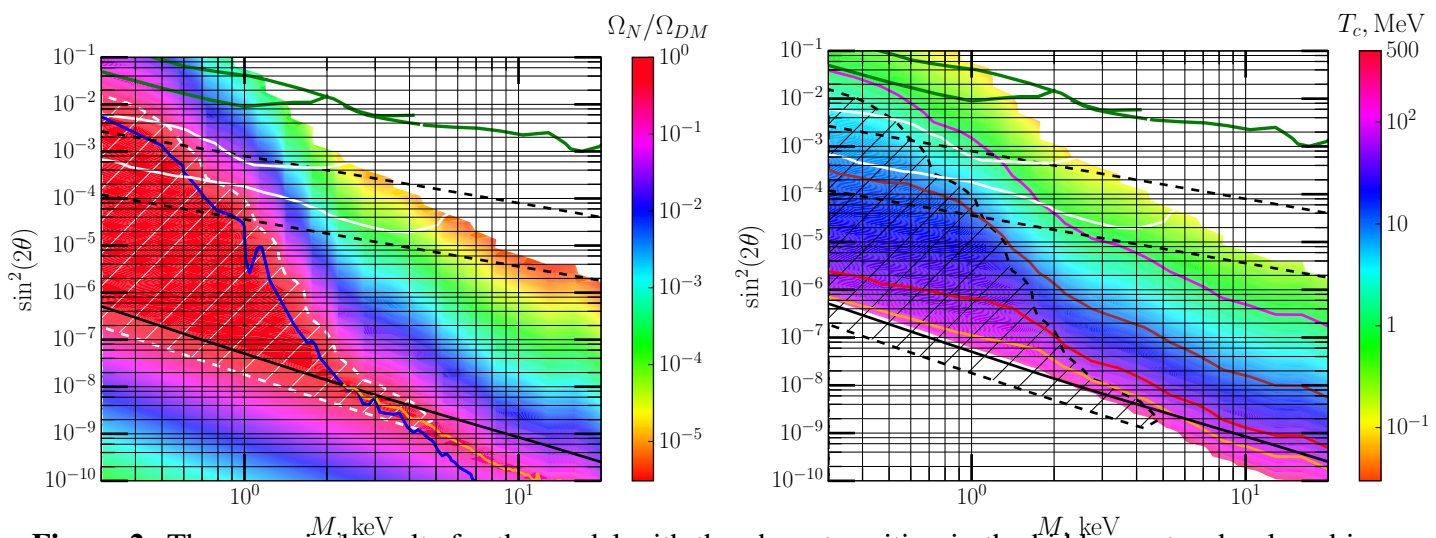

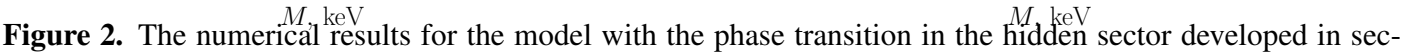
tion 3. Maximal fraction of sterile neutrinos in the total DM $\Omega_{N} / \Omega_{\mathrm{DM}}$ that satisfies the X-ray bound (14) and does not exceed the DM abundance (left panel) and corresponding phase transition temperatures $T_{c}$ (right panel) are both indicated by colour. Green curves on top of the plots represent the direct constraints from ground-based experiments [4-6]. Two solid white lines depict projected sensitivity of Troitsk $v$-mass after two stages of upgrade [18]. Inclined dashed black lines show the reference seesaw values (8) for active neutrino masses between $0.2 \mathrm{eV}$ and $0.009 \mathrm{eV}$. Black solid line on both plots corresponds to non-resonant production of $\Omega_{N}=\Omega_{\mathrm{DM}}$. In the region diagonally dashed by white (left) and black (right) lines warm sterile neutrinos constitute more than $30 \%$ of the whole DM abundance on the left and right panels, respectively, so a larger fraction of WDM is disfavoured by the structure formation constraints. Left panel: Blue solid line shows X-ray constraint $\theta_{\max }$ X-ray for $\Omega_{N}=\Omega_{\mathrm{DM}}$. Yellow solid line relates to (15). Right panel: orange, red, brown and magenta curves refer to the following temperatures of phase transition $T_{c}=100,50,10$ and $1 \mathrm{MeV}$. White region in the lower left corner denotes the area where the phase transition is not required $\left(T_{c} \rightarrow \infty\right)$.

In the region below both black and orange solid lines in Fig. 2 , or $\theta<\min \left[\theta_{\mathrm{NRP}}, \theta_{\max X-\text {-ray }}\right]$, the phase transition is not needed and $\Omega_{N}$ is just given by the usual DW quantity within the non-resonant production scenario. In the area above both blue and orange solid lines, or $\theta>\max \left[\theta_{\mathrm{NRP}}, \theta_{\mathrm{X} \text {-ray }}\right], \Omega_{N}$ saturates the bound (14). Upper right white spaces in Fig. 2 mark the forbidden region in the model of Sec. 3 where the required suppression can not be achieved due to existence of appreciable initial contribution (11) inversely proportial to $T_{c}^{2}$.

In the left-handed triangular region limited by black and blue solid lines in Fig. 2 it is possible to meet the X-ray constraint $\theta_{\mathrm{X} \text {-ray }}$ and to compose all the DM, $\Omega_{N}=\Omega_{D M}$, simultaneously, solely by reducing $T_{c}$. In fact, such a region strongly violates the structure formation tests. Indeed, even conservative bounds give in the case of non-resonantly produced DM $m_{\mathrm{NRP}}>8 \mathrm{keV}$ from Lyman- $\alpha$ [19] and of $m_{\mathrm{NRP}}>5.7 \mathrm{keV}$ [20] from phase space density. These constraints should be adopted for the sterile neutrinos of warm distribution (6) generated in the scenario of Sec. 3 by the following rescaling of the mass

$$
m_{\mathrm{NRP}}=\frac{\left.\langle\langle p\rangle\rangle\right|_{f_{N}}}{\left.\langle\langle p\rangle\rangle\right|_{f_{A}}} M=\frac{4.1}{3.1} M
$$

It leads to the actual constraint on the sterile neutrino mass $M>6 \mathrm{keV}$ and $M>4 \mathrm{keV}$, respectively, which exclude the whole region ${ }^{4}$. Actually, this kind of constraint may be irrelevant if sterile neutrinos

\footnotetext{
${ }^{4}$ Stricktly speaking, there is one more cold component (10) with average momentum $\langle\langle p\rangle\rangle=1.2 T$, but it is not sufficient to relax the structure formation bounds significantly and we do not consider it.
} 
being WDM compose only a subdominant part of the DM. Accurate investigations of this issue have been just begun in the literature ${ }^{5}$, therefore we only dashed the are where the fraction of the WDM component in the total DM is above $30 \%$ [21] by white (left) and black (right) inclined lines in left and right plots of Fig. 2, respectively. These zones indicate that the warm sterile neutrinos produced in the scenario of Sec. 3 are not able to contribute significantly to the present DM.

It is clearly from Fig. 2 that in the model of Sec. 3 it is possible to reach the direct constraints from ground-based experiments (green solid lines in Fig. 2) adopting $T_{c}>T_{c, \min }$, see (12).

It is worth noting that the upper bound of the available region (colour areas in Fig. 2) remains valid for the mixing with muon and tau neutrino species as well. Indeed, at low temperatures after electron decoupling the interaction rate in plasma has the same expression for all three active neutrinos.

Finally, in the model of Sec. 3 with the phase transition in the hidden sector neutrino oscillations in the early Universe can be strongly suppressed which provides a significant extension of available parameter space $\left(M, \theta^{2}\right)$. The presently developing ground-based experiments, see for review [18], will be able to directly probe the mixing (8) which naturally explains the generation of active neutrino masses within the seesaw type-I mechanism.

\section{Conclusions}

To summarise, we developed the model with the phase transition at some moment in the past that makes the X-ray constraints competitive with the direct laboratory searches of keV-scale sterile neutrinos.

In addition, the keV-scale sterile neutrinos with a large mixing can naturally explain active neutrino masses within the framework of the seesaw type-I mechanism. Thus, the forthcoming groundbased experiments such as Troitsk $v$-mass and KATRIN will be able to exclude this possibility in the future.

All possible scenarios are not limited by this one considered in the paper. But, generally, we need new physics at low energy scale to produce an appropriate number of sterile neutrinos with a relatively large mixing.

The works is supported by the RSF grant 17-12-01547.

\section{References}

[1] K.N. Abazajian et al. (2012), 1204.5379

[2] S. Dodelson, L.M. Widrow, Phys. Rev. Lett. 72, 17 (1994), hep-ph/9303287

[3] A.D. Dolgov, Phys. Rept. 370, 333 (2002), hep-ph/0202122

[4] K.H. Hiddemann, H. Daniel, O. Schwentker, J. Phys. G21, 639 (1995)

[5] E. Holzschuh, W. Kundig, L. Palermo, H. Stussi, P. Wenk, Phys. Lett. B451, 247 (1999)

[6] J.N. Abdurashitov et al. (2017), 1703.10779

[7] R. Fardon, A.E. Nelson, N. Weiner, JCAP 0410, 005 (2004), astro-ph/0309800

[8] R. Horvat, JCAP 0601, 015 (2006), astro-ph/0505507

[9] T. Asaka, M. Laine, M. Shaposhnikov, JHEP 01, 091 (2007), [Erratum: JHEP02,028(2015)], hep-ph/0612182

[10] K. Abazajian, G.M. Fuller, M. Patel, Phys. Rev. D64, 023501 (2001), astro-ph/0101524

\footnotetext{
${ }^{5}$ The mass dependent constraint on the available admixture of WDM in the total DM is obtained in [21]. However, these results are rather inaccurate and we do not exploit them here, simply assuming that the WDM admixture does not exceed roughly one third of the total DM in full compliance with Ref. [21].
} 
[11] J. Ghiglieri, M. Laine, JHEP 11, 171 (2015), 1506. 06752

[12] P. Minkowski, Phys. Lett. B67, 421 (1977)

[13] M. Gell-Mann, P. Ramond, R. Slansky, Conf. Proc. C790927, 315 (1979), 1306.4669

[14] R.N. Mohapatra, G. Senjanovic, Phys. Rev. Lett. 44, 912 (1980)

[15] T. Yanagida, Prog. Theor. Phys. 64, 1103 (1980)

[16] J. Schechter, J.W.F. Valle, Phys. Rev. D22, 2227 (1980)

[17] R. Adhikari et al., JCAP 1701, 025 (2017), 1602.04816

[18] D.N. Abdurashitov et al., JINST 10, T10005 (2015), 1504.00544

[19] A. Boyarsky, J. Lesgourgues, O. Ruchayskiy, M. Viel, JCAP 0905, 012 (2009), 0812 . 0010

[20] D. Gorbunov, A. Khmelnitsky, V. Rubakov, JCAP 0810, 041 (2008), 0808 . 3910

[21] R. Diamanti, S. Ando, S. Gariazzo, O. Mena, C. Weniger (2017), 1701.03128 